\title{
COMPARISONS OF THE DISTRIBUTION OF RADIOACTIVE IODINE IN SERUM AND URINE IN DIFFERENT LEVELS OF THYROID FUNCTION
}

\author{
By ROBERT H. WILLIAMS, HERBERT JAFFE, AND BEATRICE BERNSTEIN \\ (From the Thorndike Memorial Laboratory, Second and Fourth Medical Services [Harvard], \\ Boston City Hospital, the Department of Medicine, Harvard Medical School, \\ Boston, Massachusetts, and the Department of Medicine, \\ University of Washington, Seattle, Washington)
}

(Received for publication March 19, 1948)

One of the earliest uses of radioiodine was as a tool for the study of thyroid physiology (1-7). Estimations of the relative quantities of isotope stored in the thyroid have been made by means of a Geiger-Müller tube held over the gland and by determining the amount present in the urine and blood. The rate with which the level of radioiodine changes in these compartments has also been studied $(7,8)$.

In subjects with euthyroidism, radioiodine was found (8) to be excreted rapidly, within a few hours after its administration, but a plateau tended to appear after 48 hours. In myxedematous individuals, excretion was slower initially but it was more persistent, requiring four days or more; the total amount excreted after several days was greater in myxedematous subjects than in normal or thyrotoxic ones. In untreated thyrotoxic patients the rate of excretion was less than in either of the other two groups. The curves describing excretion became asymptotic relatively quickly.

The differences in the metabolism of iodine in various functional states of the thyroid have prompted an investigation of the usefulness of radioiodine as a diagnostic test. One of the best tests not dealing with radioiodine that has been available is the determination of the protein-bound iodine of the plasma (9). This test is so long and difficult that it can be performed satisfactorily only under the direction of a few highly qualified individuals and even then some overlapping in the values for different degrees of thyroid function may be found (10). Moreover, the test is unreliable if the patient has recently received iodine in organic form and if highly scrupulous technique is not followed in the collection of the plasma as well as in the determination. It seemed to us that in some institutions it might be possible to use radioiodine in tests which would be more accurate, simpler and more rapid. It appears logical to assume that the quantity of protein-bound radioiodine in the serum, after the administration of tracer doses, might indicate the relative rate of manufacture of the thyroid hormone and its release into the blood stream. Of course, the values obtained would represent only an approximate balance between these two factors and the quantity of the hormone stored in the various fluids and tissues of the body, including the thyroid; also between the amount metabolized and excreted. Nevertheless, with methods not using the radioiodine, not only do these difficulties in interpretation exist, but also it is not possible to determine the time required for the changes to occur. The latter aspect would seem to be a very important one.

\section{METHODS}

Two types of study were conducted following the administration of a tracer dose of radioiodine subcutaneously. In one, frequent specimens of blood and urine were collected during a 24-hour interval, for a determination of the proportion of isotope present. In the other study, which consisted of a larger number of patients, urine was saved for 24 hours and a single specimen of blood was taken at the end of this interval. $I^{131}$ obtained from Clinton Laboratories, Oak Ridge, Tennessee, was used for the studies; no carrier was added.

Determination of the radioiodine in the specimens was by the methods previously described (11). Trichloracetic acid was used for precipitation of protein-bound isotope. The "DI" (diiodotyrosine iodide) fraction was separated from the "TI" (thyroxin iodide) by butyl alcohol precipitation (9).

In the first experiment there were seven individuals with euthyroidism, four with thyrotoxicosis, three with athyreosis and one with Addison's disease. The patients with thyrotoxicosis had had no treatment. Those with athyreosis were in a state of euthyroidism as a result of therapy with desiccated thyroid, thereby affording an opportunity to observe the effects of the thyroid upon 
certain aspects of iodine metabolism. Results obtained in patients so treated would be expected to be unlike those of untreated patients in that the metabolic rate would be higher in the former group, but the two types are similar in that in both instances essentially no thyroxin can be synthesized by the thyroid. The subject with Addison's disease was adequately treated with desoxycorticosterone. Each of the patients in this experiment was hospitalized while the studies were conducted.

In the second experiment there were 20 subjects with euthyroidism, eight with athyreosis receiving treatment with desiccated thyroid, 17 with definite thyrotoxicosis, and seven with questionable thyrotoxicosis. The group with euthyroidism consisted of ten internes, two nurses, and eight patients with a variety of non-thyroid diseases, none of which were severe. Ten of the thyrotoxic patients had had no previous therapy; the others had had propylthiouracil but none for four days or more preceding the test, and it was found that the results were similar to those without previous therapy. Two of the seven subjects with questionable thyrotoxicosis had been treated previously with propylthiouracil, but had not received any for more than one week preceding the radioiodine. Each of the seven patients had a goiter. Very few of the subjects in this experiment were hospitalized.

\section{RESULTS}

The results of the first experiment are shown in Figure 1. The subjects with euthyroidism and the ones with athyreosis excreted radioiodine in the urine much more rapidly than did the patients with the thyrotoxicosis. None of the latter group excreted as much as 20 per cent of the injected dose within the first 24 hours, whereas each of the other two groups excreted distinctly more than this quantity. The myxedematous subjects had excreted essentially the same quantity of isotope as normals by the end of four hours. In the patient with Addison's disease, the excretion of isotope in the urine and its concentration in the

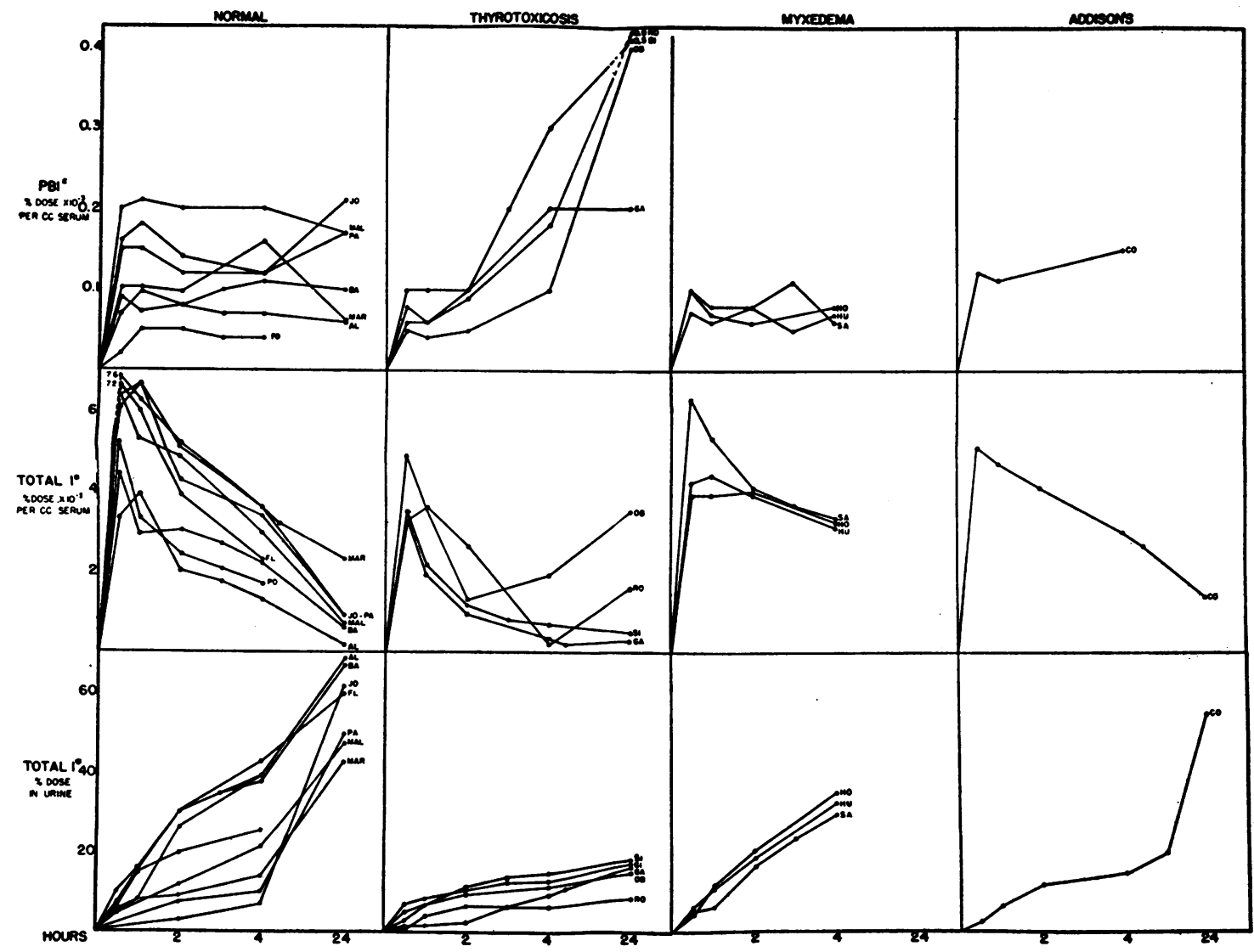

FIG. 1

Each patient was given 100 microcuries of radioiodine ( $\left.I^{121}\right)$ subcutaneously without any carrier. PBI* indicates the protein-bound radioiodine. All values are expressed with reference to the dose of isotope administered. 
serum were within normal range. During the first four hours the concentration of total radioiodine in the serum of thyrotoxic patients tended to be lower than in the subjects with euthyroidism or athyreosis. It can be observed in Figure 1 that two of the thyrotoxic patients had a second rise in the concentration of isotope. The quantity of protein-bound radioiodine rapidly reached a pla-

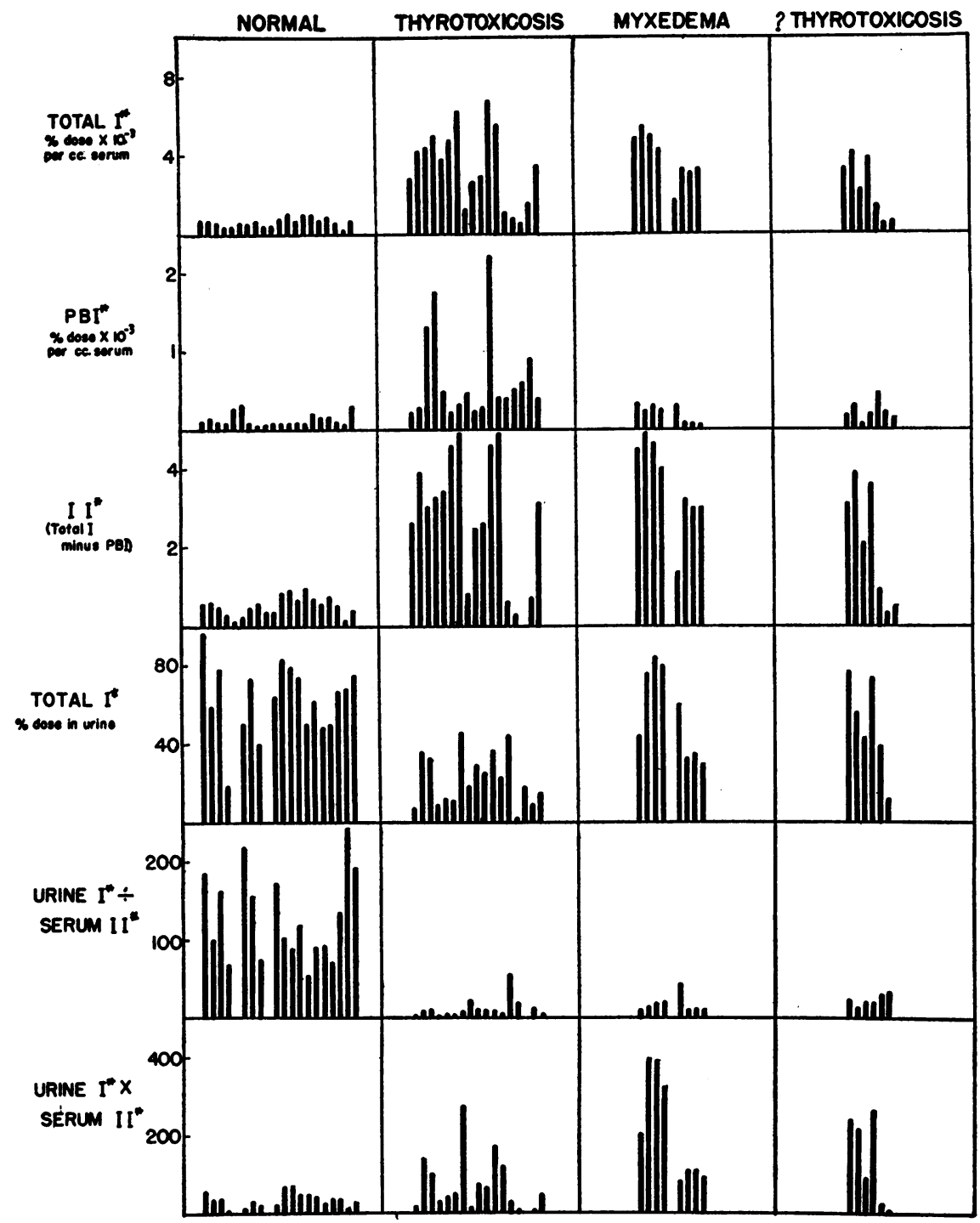

FIG. 2

The serum specimens were obtained 24 hours following the administration of radioiodine; during this interval all urine was saved for analysis; two patients neglected to collect urine. The quantity of radioiodine in inorganic (II*) form in the serum was derived by subtracting the protein-bound radioiodine (PBI*) from the total radioiodine content of serum. The reasons for empirically dividing and multiplying the values for the radioiodine in urine by the inorganic radioiodine in the serum are discussed in the text. The data shown in the seventh column of the group with "? thyrotoxicosis" are those of a patient who was subsequently shown not to have thyrotoxicity. Unfortunately, this patient failed to save all of his urine. The diagnosis of the individual with data presented in the sixth column is still questionable. In four patients myxedema was primary and in the other four (last four columns) it was secondary. 


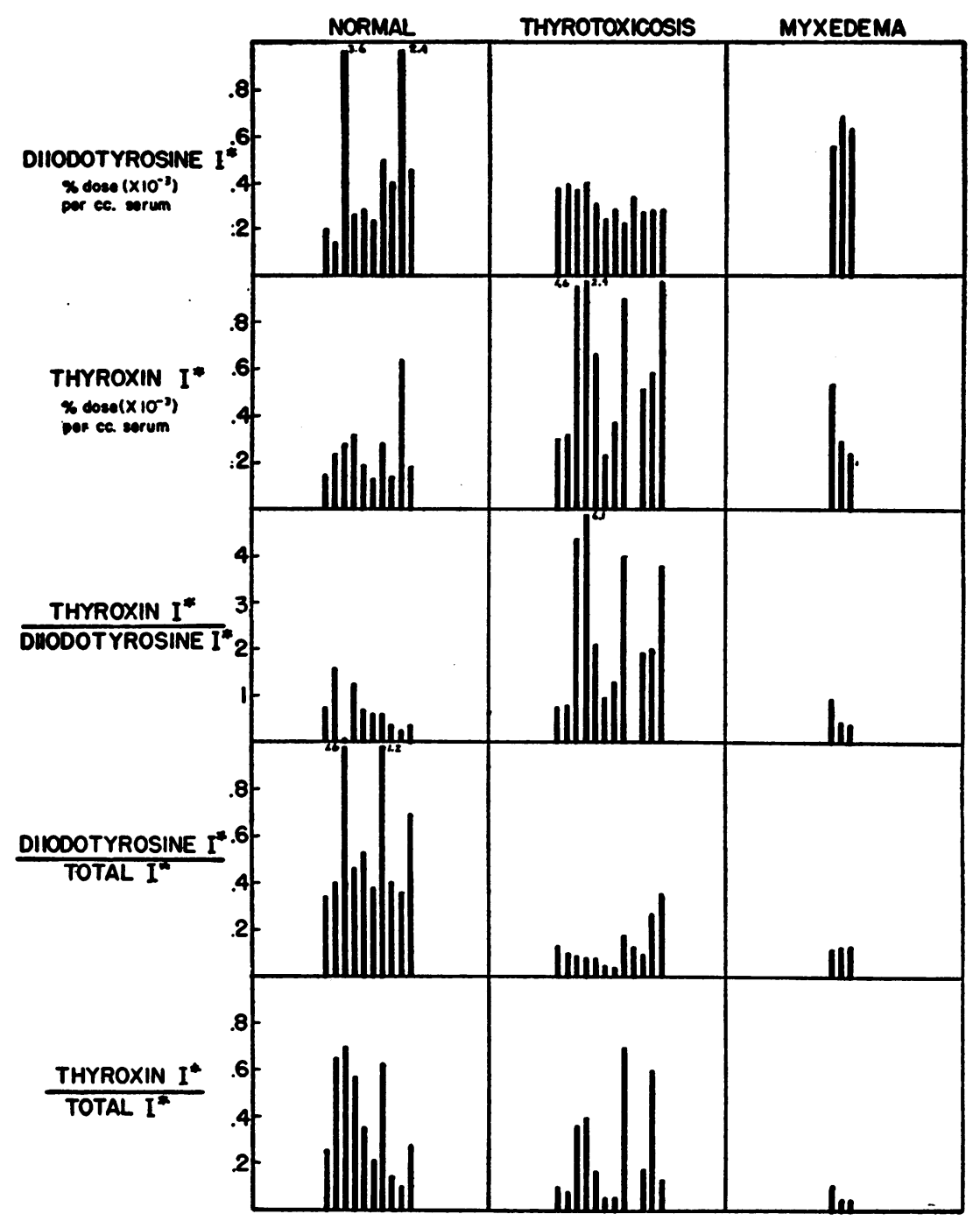

Fig. 3

These data were collected upon some of the patients represented in Figure 2.

teau in the individuals with euthyroidism and athyreosis. In the ones with thyrotoxicosis the increase was slow in the first few hours, but after 24 hours it was markedly higher in three of the four thyrotoxics than in any other subject. These observations prompted the performance of the second experiment, the results of which are shown in Figure 2.

The concentration in the serum of total radioiodine was distinctly greater in the patients with thyrotoxicosis than it was in normal individuals. In considering these findings in conjunction with the subnormal excretion of radioiodine in the urine within a 24-hour interval, it must be borne in mind, as illustrated in Figure 1, that during several hours the concentration of total radioiodine in the serum was less in the thyrotoxic subjects than in normals. Moreover, in the former group, during the latter part of the 24-hour interval more of the radioiodine was protein-bound and thus less available for excretion.

The concentration of total and inorganic radioiodine serum of myxedematous patients was greater than normal, while the protein-bound radioiodine of the serum and the quantity of isotope in the urine were essentially normal. As to why there should be the discrepancies in the ratios of inorganic iodine in the serum and the quantity in 
the urine, the answer is not clear. However, we attempted to emphasize these discrepancies in the different groups by empirically dividing the urine values by the serum inorganic iodine, and, also by multiplying them. As seen in Figure 2, there is a marked difference in the ratio of urine to serum values in the normal group as compared to the others. The product of the values is distinctly greater than normal in each of the subjects with athyreosis; it is also greater in some, but not all, of the thyrotoxic patients.

In serum of some of the patients the diiodotyrosine radioiodine and thyroxin radioiodine fractions were determined (Figure 3). No very striking difference in these fractions was observed in the various groups. The ratio of thyroxin to diiodotyrosine tended to be somewhat greater in the thyrotoxic patients, but there is too much overlapping of values to be of practical significance. On the other hand, the ratio of diiodotyrosine radioiodine to total radioiodine is definitely greater in the normal subjects than in the others. The tendency in normals for a greater ratio of thyroxin radioiodine to total radioiodine was an unexpected finding.

\section{DISCUSSION}

All of the indirect methods that have been proposed for the evaluation of the functional state of the thyroid gland by the determination of its uptake of radioiodine are associated with certain inaccuracies. Among these are the variations in thickness and contour of the neck, innumerable factors affecting kidney function, differences in the volume of the fluid compartments, and probably the rate of metabolism of the thyroid hormone and of its synthesis and concentration in non-thyroid tissues. Although Hertz, Hamilton and Soley and their colleagues (1-5), respectively, found that patients with hyperthyroidism accumulated a larger proportion of the tracer dose in the thyroid than did normal subjects, Astwood and Stanley (12) found that the concentration gradients of the group with thyrotoxicosis was not significantly above the normal range. The failure of the latter investigators to administer any carrier iodine might account for some of the difference in results. Whereas some investigators $(3,4)$ have measured the excretion of radioiodine in the urine as an index to the quantity retained in the thyroid gland, a poor correlation has been found (13) between determinations of this type and direct measurements over the thyroid. However, all of the other clinical tests of thyroid function are beset with diffculties.

On the basis of data which we have presented, as well as those of others, determinations of the proportion of a tracer dose excreted in the urine are of diagnostic importance, but are not infallible. The measurements of the total radioiodine concentration in the serum, or of the measurements of the protein-bound thyroxin and diiodotyrosine radioiodine components did not demonstrate as clear a differentiation of the various levels of thyroid function as was desired. Nevertheless, it is believed that these estimations and certain ones of the ratios, that have been discussed, may be of some significance. It seems likely that greater differentiations in the protein-bound radioiodine components could be demonstrated if longer intervals were permitted to elapse before collecting the specimens, or by following the rate of the changes.

\section{SUMMARY}

Studies were conducted using radioiodine for the evaluation of different degrees of thyroid function, as found in subjects with thyrotoxicosis, athyreosis, or euthyroidism. Following the administration of 100 microcuries of radioiodine $\left(\mathrm{I}^{131}\right)$ carrier free, it was confirmed that thyrotoxic patients excreted less of the isotope in the urine than did the other groups. Although the concentration in the serum of total radioiodine tended to be less in the thyrotoxic group than in the others, within the first few hours, after 24 hours the total radioiodine and its protein-bound, thyroxin, and inorganic radioiodine components tended to be supernormal. In the group with athyreosis the total and inorganic radioiodine concentrations were supernormal. The ratio of diiodotyrosine radioiodine to thyroxin radioiodine, as well as a somewhat empirically calculated ratio of urine radioiodine and serum inorganic radioiodine, was distinctly less in the patients with thyrotoxicosis or athyreosis than in the normals. The product of the radioiodine of the urine and of the inorganic form of the serum was distinctly greater in the subjects with athyreosis than in normals and tended to be 
greater in the former group than in the ones with thyrotoxicosis.

There was no good correlation of the results with the various degrees of thyrotoxicity.

Tests of the type reported, like all of the others of thyroid function, should be considered in conjunction with the clinical findings.

\section{BIBLIOGRAPHY}

1. Hertz, S., Roberts, A., and Evans, R. D., Radioactive iodine as indicator in the study of thyroid physiology. Proc. Soc. Exper. Biol. \& Med., 1938, 38, 510.

2. Hertz, S., Radioactive iodine as an indicator in thyroid physiology. Observations on rabbits and on goiter patients. Am. J. Roentgenol., 1941, 46, 467.

3. Hertz, S., Roberts, A., and Salter., W. T., Radioactive iodine as an indicator in thyroid physiology. IV. The metabolism of iodine in Graves' disease. J. Clin. Invest., 1942, 21, 25.

4. Hamilton, J. G., and Soley, M. H., Studies in iodine metabolism by the use of a new radioactive isotope of iodine. Am. J. Physiol., 1939, 127, 557.

5. Hamilton, J. G., and Soley, M. H., Studies in iodine metabolism of thyroid gland in situ by use of radioiodine in normal subjects and in patients with various types of goiter. Am. J. Physiol., 1940, 131, 135.

6. Rawson, R. W., Evans, R. D., Means, J. H., Peacock, W. C., Lerman, J., and Cortell, R. E., The action of thiouracil upon the thyroid gland in Graves' disease. J. Clin. Endocrinol., 1944, 4, 1.

7. Rawson, R. W., and McArthur, J. W., Radioiodine, its use as a tool in the study of thyroid physiology. J. Clin. Endocrinol., 1947, 7, 235.

8. Keating, F. R., Jr., Power, M. H., Berkson, J., and Haines, S. F., The urinary excretion of radioiodine in various thyroid states. J. Clin. Invest., 1947, 26, 1138.

9. Salter, W. T., The Endocrine Function of Iodine. Harvard University Press, Cambridge, Mass., 1940.

10. Winkler, A. W., Disorders of the Thyroid Gland, in: Diseases of Metabolism, edited by Duncan, G. G. W. B. Saunders Co., Philadelphia and London, 1947, pp. 896-938.

11. Williams, R. H., Jaffe, H., Towery, B. T., Rogers, W. F., and Tagnon, R., Factors influencing the effectiveness of radioiodotherapeusis. In press.

12. Astwood, E. B., and Stanley, M. M., The use of radioactive iodine in the study of thyroid function in man. West. J. Surg., 1947, 55, 625.

13. Quimby, E. H., and McCune, D. H., cited by Astwood and Stanley (12). 